\title{
Timing of PMMA cement application for pedicle screw augmentation affects screw anchorage
}

\author{
Werner Schmoelz $^{1}$ - Christian Heinz Heinrichs ${ }^{1} \cdot$ Sven Schmidt $^{2} \cdot$ Angel R. Piñera $^{3}$ • \\ Felix Tome-Bermejo ${ }^{3} \cdot$ Javier M. Duart $^{3} \cdot$ Marlies Bauer $^{4} \cdot$ Luis Álvarez Galovich $^{3}$
}

Received: 25 October 2016/Revised: 13 March 2017/Accepted: 17 March 2017/Published online: 3 April 2017

(C) The Author(s) 2017. This article is an open access publication

\begin{abstract}
Introduction Cement augmentation is an established method to increase the pedicle screw (PS) anchorage in osteoporotic vertebral bodies. The ideal timing for augmentation when a reposition maneuver is necessary is controversial. While augmentation of the PS before reposition maneuver may increase the force applied it on the vertebrae, it bears the risk to impair PS anchorage, whereas augmenting the PS after the maneuver may restore this anchorage and prevent early screw loosening. The purpose of the present study was to evaluate the effect of cement application timing on PS anchorage in the osteoporotic vertebral body.

Methods Ten lumbar vertebrae (L1-L5) were used for testing. The left and right pedicles of each vertebra were instrumented with the same PS size and used for pairwise comparison of the two timing points for augmentation. For the reposition maneuver, the left PS was loaded axially under displacement control $(2 \times \pm 2 \mathrm{~mm}, 3 \times \pm 6 \mathrm{~mm}$, $3 \times \pm 10 \mathrm{~mm}$ ) to simulate a reposition maneuver.
\end{abstract}

Werner Schmoelz

werner.schmoelz@i-med.ac.at

Christian Heinz Heinrichs

Christian.Heinrichs@i-med.ac.at

Sven Schmidt

s.schmidt@ friedrichsheim.de

Angel R. Piñera

ARpineraP@fjd.es

Felix Tome-Bermejo

felixtome@hotmail.com

Javier M. Duart

javier.duart@uv.es

Marlies Bauer

Marlies.bauer@i-med.ac.at
Subsequently, both PS were augmented with 2 ml PMMA cement. The same force as measured during the left PS maneuver was applied to the previously augmented right hand side PS $[2 \times F( \pm 2 \mathrm{~mm}), 3 \times F \quad( \pm 6 \mathrm{~mm})$, $3 \times F( \pm 10 \mathrm{~mm})]$. Both PS were cyclically loaded with initial forces of +50 and $-50 \mathrm{~N}$, while the lower force was increased by $5 \mathrm{~N}$ every 100 cycles until total failure of the PS. The PS motion was measured with a 3D motion analysis system. After cyclic loading stress, X-rays were taken to identify the PS loosening mechanism.

Results In comparison with PS augmented prior to the reposition maneuver, PS augmented after the reposition maneuver showed a significant higher number of load cycles until failure $(5930 \pm 1899$ vs $3830 \pm 1706$, $p=0.015$ ). The predominant loosening mechanism for PS augmented after the reposition maneuver was PS toggling with the attached cement cloud within the trabecular bone. While PS augmented prior to the reposition, maneuver showed a motion of the screw within the cement cloud.

Luis Álvarez Galovich

Lalvarez@fjd.es

1 Department of Trauma Surgery, Medical University of Innsbruck, Anichstrasse 35, 6020 Innsbruck, Austria

2 Orthopaedic University Hospital Friedrichsheim, Frankfurt am Main, Germany

3 Spine Service, Fundacion Jimenez Diaz University Hospital, Madrid, Spain

4 Division of Clinical and Functional Anatomy, Department of Anatomy, Histology and Embryology, Medical University of Innsbruck, Innsbruck, Austria 
Conclusion The time of cement application has an effect on PS anchorage in the osteoporotic vertebral body if a reposition maneuver of the instrumented vertebrae is carried out. PS augmented after the reposition maneuver showed a significant higher number of load cycles until screw loosening.

Keywords Pedicle screws - Augmentation - Osteoporosis · Reposition maneuver · Biomechanics .

Polymethylmethacrylate

\section{Introduction}

Osteoporosis is a disease characterized by decreased bone strength, increased skeletal fragility, and fracture susceptibility. In the European Union, over 27 million individuals currently suffer from osteoporosis, and due to the demography change, this number will increase by $23 \%$ within the next 10 years [1]. Osteoporosis predisposes these patients to progressive spinal deformities and potential neurologic compromise from vertebral compression fractures. Surgical intervention with instrumentation for the osteoporotic spine is sometimes a clinical necessity. The gold standard to surgically stabilize the spine is pedicle screw fixation. During the surgical procedure, placed pedicle screws are also used to conduct reposition maneuvers for fracture reduction or for deformity correction. In patients with poor bone stock, the anchorage of pedicle screws in the vertebral body is reduced and reposition maneuvers can further compromise the screw anchorage.

Pedicle screw augmentation with polymethylmethacrylate (PMMA) cement enhances initial fixation or resistance to loosening under dynamic loading conditions of the screws in osteoporotic bone [2-7]. However, the ideal timing for augmentation when a reposition maneuver is necessary, such as in fractures or deformities, is still controversially discussed. While top loading pedicle screws must be augmented prior to final construct fixation, side loading pedicle screws (rod fixation at the side of the long screw axis) give the option of in situ augmentation before or after complete construct assembly. Augmentation of the pedicle screws prior to the reposition maneuver will likely increase the force that can be applied during the maneuver. However, it bears the risk to impair the anchorage of pedicle screws already during the maneuver. On the other hand augmentation of the pedicle screws after the reposition maneuver might result in trabecular bone fractures during the maneuver. However, reduced screw anchorage due to trabecular fractures caused during the reposition maneuver can be compensated and even enhanced by subsequent augmentation.

The purpose of the present study was to investigate if timing of the cement application for augmented pedicle screw constructs subjected to a reposition maneuver is affecting pedicle screw anchorage. It was hypothesized that pedicle screws augmented after the reposition maneuver will resist a higher number of load cycles until failure of the construct and will have a smaller per cycle motion during loading than pedicle screws augmented prior to the reposition maneuver.

\section{Methods}

\section{Specimens}

Ten fresh frozen lumbar vertebrae (L1-L5) from two donors were used for testing (m, f age 69 and 91). The bodies were donated by people who had given their informed consent for their use for scientific and educational purposes prior to death $[8,9]$. Trabecular bone mineral density (BMD) of the specimens was measured using quantitative computed tomography (qCT, Lightspeed VCT16, GE Healthcare, Milwaukee, WI, USA) including a calibration phantom (EFP, European Forearm Phantom). Only osteoporotic specimens (mean BMD $59.6 \mathrm{mg} \mathrm{HA} /$ $\mathrm{ccm} \pm 20.8$ ) with a pedicle size to fit $6.35 \times 50 \mathrm{~mm}$ pedicle screws were chosen for testing.

For preparation, soft tissues such as muscle and fat were dissected. The single vertebral bodies were embedded in PMMA (Technovit 3040, Heraeus Kulzer GmbH, Wehrheim, Germany) to form an endplate mold for fixation purpose in the testing machine. Specimens were double shrink-wrapped and frozen at $-20{ }^{\circ} \mathrm{C}$ and were thawed overnight at $6{ }^{\circ} \mathrm{C}$ before testing.

Standard instruments were used for implantations. Fenestrated and side-loaded pedicle screws (Omega-21-LP pedicle screw, $6.35 \times 50 \mathrm{~mm}$, Biomet Spain, Valencia, Spain) with PMMA cement (Biomet bone cement V, Biomet Orthopaedics, Dietikon, Switzerland) were used.

\section{Instrumentation}

Left and right pedicles of the same vertebrae were instrumented with the same size pedicle screws and were used for pairwise comparison of the two augmentation timepoints. The two test groups made up of the left and right pedicles of each tested specimen (Fig. 1).

First, the reposition maneuver was carried out on the left, non-augmented pedicle screw (Man_Aug). After the reposition maneuver, the left pedicle screw was augmented with $2.0 \mathrm{ml}$ of PMMA cement. The cement was injected 4 min after cement mixing and was left for cement curing at room temperature for $120 \mathrm{~min}$.

The right pedicle screw was augmented with $2.0 \mathrm{ml}$ of PMMA cement immediately after implantation 


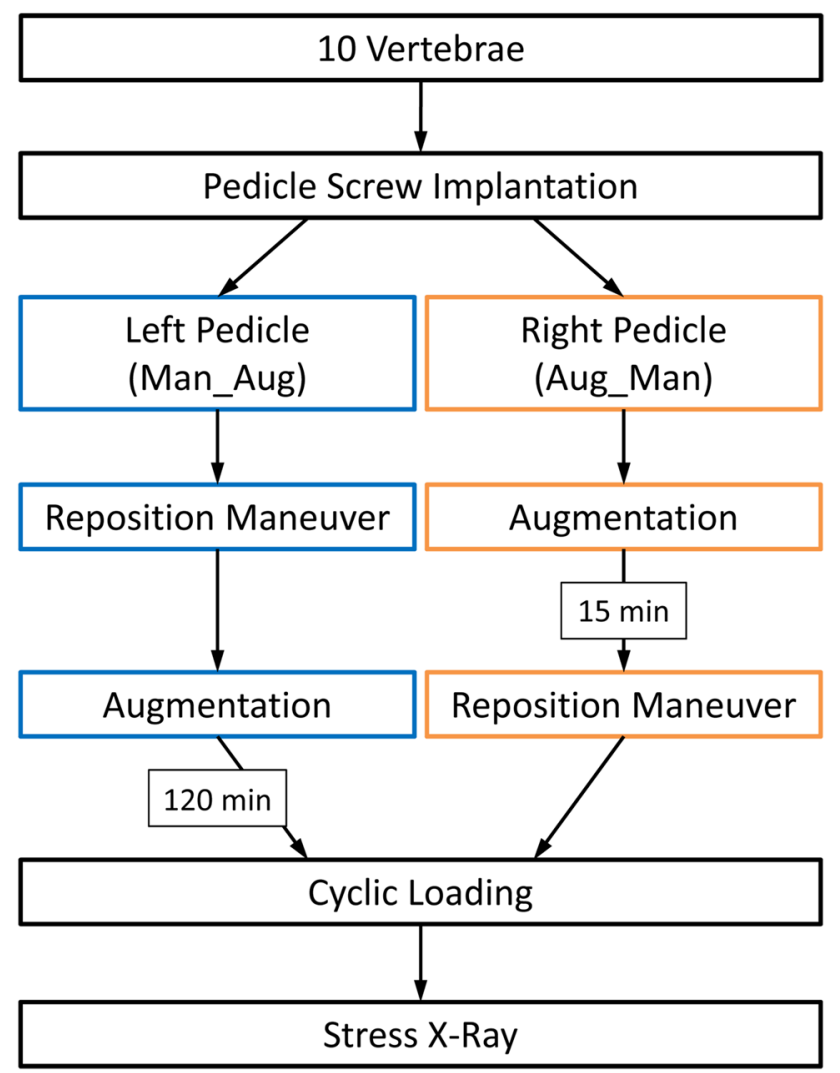

Fig. 1 Experimental procedure for the two test groups

(Aug_Man). PMMA cement injection was started $4 \mathrm{~min}$ after mixing of the PMMA cement, and the reposition maneuver at the right pedicle screw was carried out $15 \mathrm{~min}$ after cement mixing with the cement not being fully cured.
Prior to cyclic loading, an axial (crano-caudal) X-ray was taken to verify that the cement clouds around both screws were not connected.

\section{Reposition maneuver}

The reposition maneuver was carried out in a servohydraulic biaxial material testing machine (Mini-Bionix 858; MTS, Eden Prairie, MN, USA) with a lever arm of $100 \mathrm{~mm}$ connected to the pedicle screw. The specimen was fixed to an $\mathrm{x}-\mathrm{y}$ bearing table to allow translation during the reposition maneuver. To incorporate the lever arm applied by the surgeon, an extension rod was fixed to the pedicle screw and a bearing eye rigidly attached to the specimen fixation acted as fulcrum. The bearing eye was positioned on the extension rod as closely as possible to the pedicle and allowed rotation and translation in the axis of the extension rod, as well as angular tilt of the bearing eye (Fig. 2).

First, the reposition of the left, non-augmented screw (Man_Aug) was carried out with a speed of $1 \mathrm{~mm} / \mathrm{s}$ in three different displacement magnitudes (upper and lower limit) of the machine actuator with varying cycles $[2 \times \pm 2 \mathrm{~mm}, 3 \times \pm 6 \mathrm{~mm}$, and $3 \times \pm 10 \mathrm{~mm}$ displacement]. The force $\left(F_{\min }\right.$ and $\left.F_{\max }\right)$ for $\pm 2, \pm 6$, and $\pm 10 \mathrm{~mm}$ displacement was recorded.

The previously augmented right pedicle screw (Aug_Man) in the same vertebra was loaded with a speed of $1 \mathrm{~mm} / \mathrm{s}$ to force magnitudes measured during the reposition of the contralateral non-augmented pedicle screw (Man_Aug) $[2 \times F \quad( \pm 2 \mathrm{~mm}), 3 \times F \quad( \pm 6 \mathrm{~mm})$, and $3 \times F( \pm 10 \mathrm{~mm})]$.
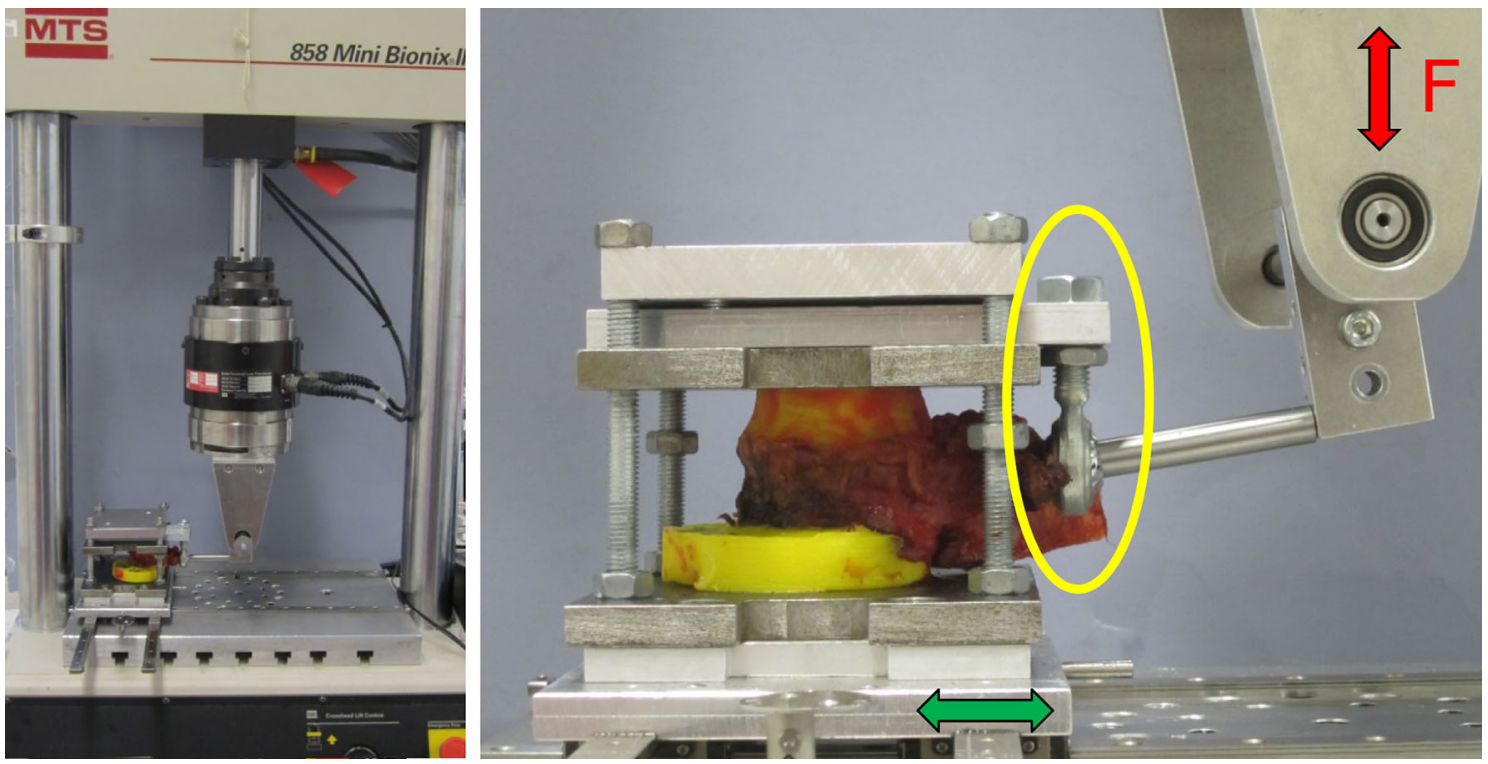

Fig. 2 Setup for the reposition maneuver in the material testing machine (left). On the right hand side the load application (red arrow) and the bearing eye (yellow) and the possible translation (green arrow) during the reposition maneuver are shown (PMMA embedding in yellow) 


\section{Cyclic loading}

The instrumented specimen was fixed in the servohydraulic biaxial material testing machine to an $\mathrm{x}-\mathrm{y}$ bearing table allowing translation in the anterior-posterior as well as the medial-lateral plane during the cyclic loading (Fig. 3). The loading rod was connected to the pedicle screw with a lever arm of $15 \mathrm{~mm}$ to the rotational axis [2].

A 3D motion analysis system (Zebris, Winbiomechanics, Isny, Germany) was mounted to the screw head and the $\mathrm{x}-\mathrm{y}$ bearing table. The pedicle screw was loaded with a cyclic load initially ranging from +50 to $-50 \mathrm{~N}$. The lower force limit was increased by $5 \mathrm{~N}$ after every 100th cycle until total failure of the pedicle screw. The material testing machine was programmed in displacement control $(5 \mathrm{~mm} / \mathrm{s})$ with force limits. During testing, the screw head motion relative to the base plate was recorded with the $3 \mathrm{D}$ motion analysis system every 100th cycle. Cyclic loading was terminated after $10 \mathrm{~mm}$ of machine actuator displacement relative to the unloaded starting point. For post testing data analysis, the failure criteria were defined as an absolute angular tilt of more than $8^{\circ}$ or an angle amplitude increase of more than $1^{\circ}$ within 100 cycles [2]. Per cycle motion of the screw within the vertebral body was evaluated after 1000, 2000, and 3000 cycles.

\section{Stress X-rays}

After cyclic loading, each vertebral body was cut in the sagittal plane midline using a handsaw. A rod was connected to the pedicle screw and loaded alternately in caudal and cranial directions with $20 \mathrm{~N}$, and X-rays were taken in both extreme points (caudal and cranial direction) to document the screw position and to identify the pedicle screw loosening mechanism.

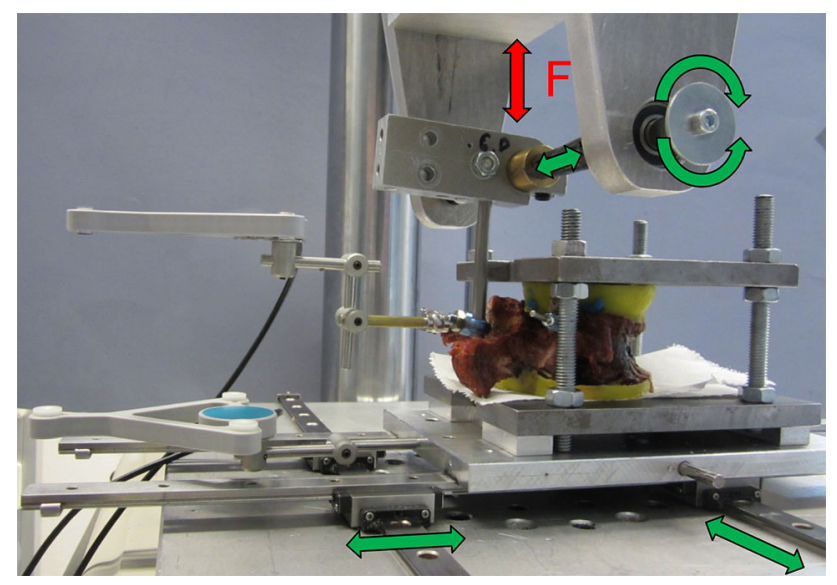

Fig. 3 Test setup for cyclic loading in the material testing machine. Green arrows indicate the possible motions of the setup during cyclic loading (red arrow)

\section{Statistical analysis}

Statistical analysis was carried out using the SPSS software package 22.0 (IBM Corporation, Armonk, NY, USA). Kolmogorow-Smirnow tests were performed for evaluating the distribution pattern. As all data were normal distributed, paired $t$ tests were applied for comparison of the augmentation techniques of the left and right pedicle screws. The test protocol was completed for all vertebrae and all specimens could be included in the statistical analysis.

\section{Results}

Mean tensile and compressive forces measured at the machine actuator during the reposition maneuver were 104.6 $\mathrm{N} \pm 43.6$ and $-95 \mathrm{~N} \pm 34.71$ for $\pm 2 \mathrm{~mm}$ displacement, $343.2 \mathrm{~N} \pm 87.9$ and $-245.2 \mathrm{~N} \pm 47.9$ for $\pm 6 \mathrm{~mm}$ displacement, and $470.7 \mathrm{~N} \pm 170.6$ and $-358.8 \mathrm{~N} \pm 87.3$ for $\pm 10 \mathrm{~mm}$ displacement.

\section{Cyclic loading}

For the left pedicle screws (Man_Aug), the mean failure cycle (load) was significant higher $(p=0.015)$ than for the right pedicle screws (Aug_Man), $5930 \pm 1899$ vs $3830 \pm 1706$, respectively (Fig. 4). With the increasing load magnitude, these failure load cycles correspond to a mean loading of $345 \mathrm{~N}$ for the Man_Aug group and $240 \mathrm{~N}$ for the Aug_Man group.

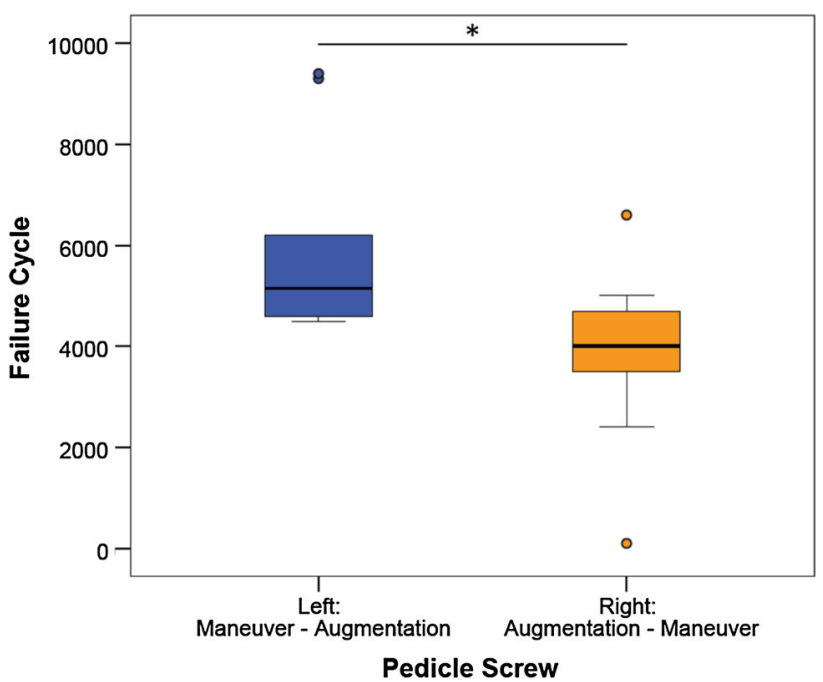

Fig. 4 Number of load cycles to failure during the cyclic loading with stepwise increasing load magnitude for the two augmentation techniques. Boxplots showing medians and interquartile ranges $(25-75 \%)$. Asterisk indicates significant differences $(p<0.05)$ 


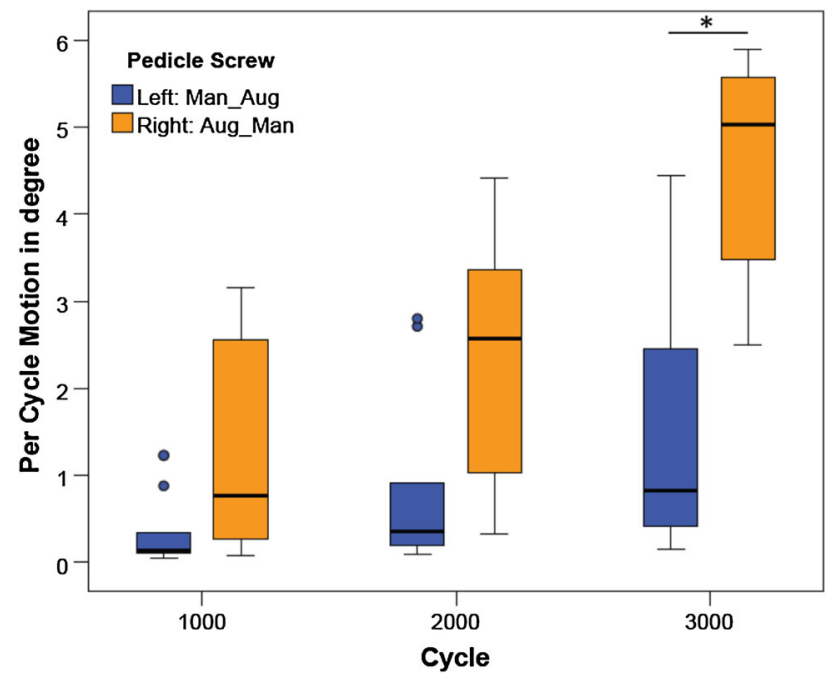

Fig. 5 Per cycle motion of the two augmentation time points after 1000, 2000 and 3000 cycles. Boxplots showing medians and interquartile ranges $(25-75 \%)$. Asterisk indicates significant differences $(p<0.05)$
The mean per cycle motion of the left pedicle screw (Man_Aug) after 1000, 2000, and 3000 cycles was lower than for the right pedicle screw (Aug_Man) (Fig. 5). After 1000 load cycles, this difference was not significant $\left(p=0.160 ; 0.36^{\circ} \pm 0.42^{\circ}\right.$ vs $\left.1.30^{\circ} \pm 1.27^{\circ}\right)$, after 2000 load cycles, this difference showed a trend $(p=0.085$; $0.92^{\circ} \pm 1.09^{\circ}$ vs $\left.2.33^{\circ} \pm 1.47^{\circ}\right)$, while it was significant after 3000 cycles $\quad\left(p=0.018 ; \quad 1.70^{\circ} \pm 1.69^{\circ} \quad\right.$ vs $\left.4.57^{\circ} \pm 1.27^{\circ}\right)$.

\section{Loosening mechanism}

Left pedicle screws augmented after to the reposition maneuver (Man_Aug) showed toggling of the pedicle screws with the attached PMMA cement cloud within the trabecular bone as predominant loosening mechanism. Six of ten pedicle screws did not move within the cement cloud (Fig. 6, left).

The predominant loosening mechanism for the right pedicle screws augmented prior the reposition maneuver
Fig. 6 Example for the loosening mechanism of the two augmentation techniques. Motion of the cement cloud attached to the screw in the vertebral body (left) and motion of the screw within the cement cloud (right)

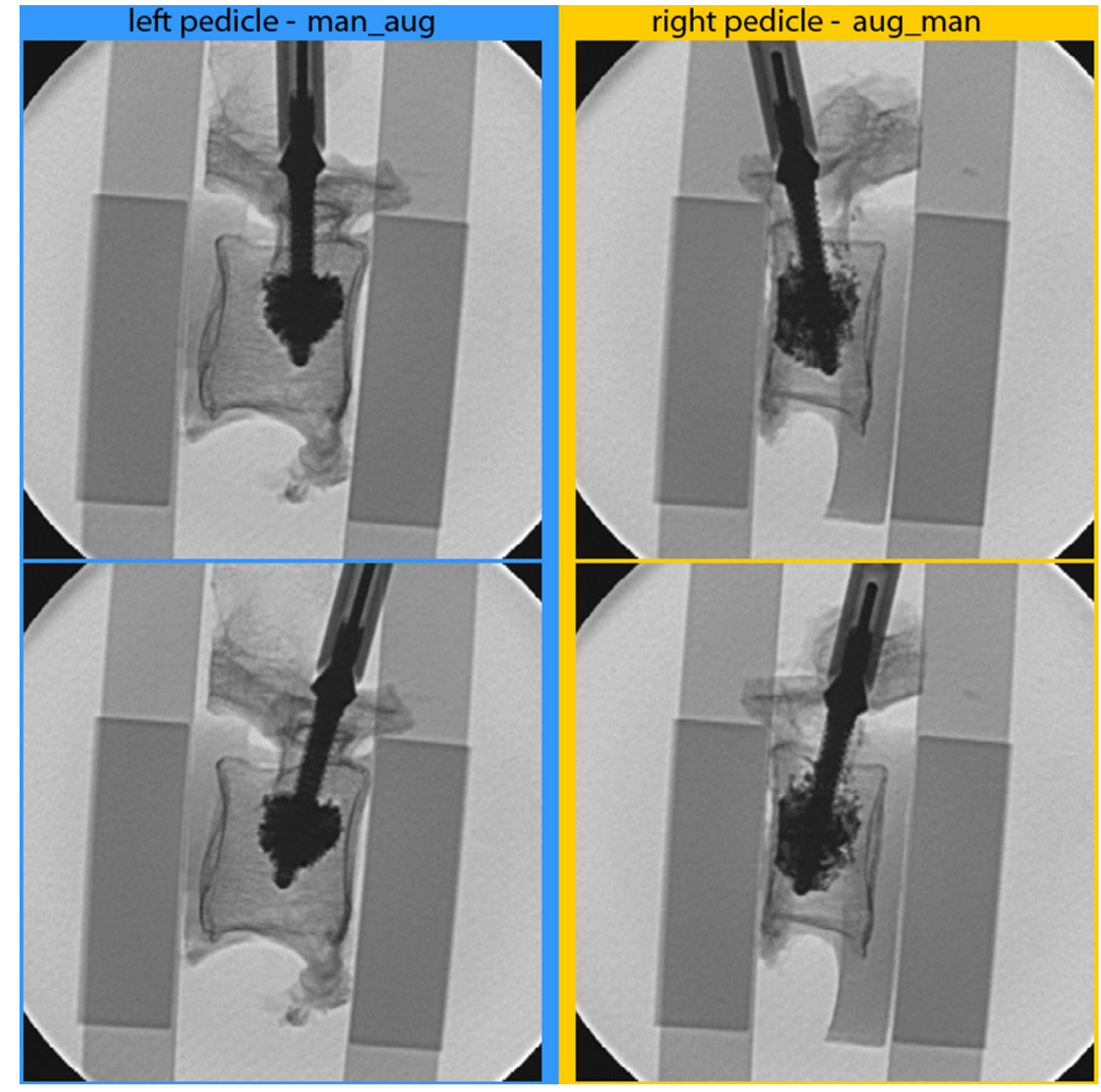


(Aug_Man) was pedicle screw motion within the cement cloud (Fig. 6, right) without cement movement within the trabecular bone of the vertebral body (9/10 specimens).

\section{Discussion}

In recent years, fracture and implant fixation in the elderly patients with reduced bone quality gained more and more attention. New concepts range from alternative fixations options such as transfacet screws or lamina hooks $[10,11]$ to expandable or modified pedicle screws $[12,13]$ and to bone cements with modified, more physiological mechanical properties [14, 15]. For conventional pedicle screws, various augmentation techniques and augmentation materials to prevent screw loosening are available [16]. So far, the augmentation of pedicle screws with PMMA has demonstrated to be the most efficient solution to enhance screw strength in osteoporotic bone [2-7]. It has shown clinical efficacy in the management of degenerative disease [17] and fractures [18-20]. However, there is a concern about the ideal time for augmentation during the surgical procedure, especially if a restoration or a derotation maneuver of the instrumented vertebra is part of the surgery.

The results of the present study have demonstrated that if a reposition maneuver or derotation is conducted, the time of cement application has an effect on pedicle screw anchorage in the osteoporotic vertebral body. Therefore, both hypotheses can be confirmed.

In comparison with pedicle screws augmented prior to the reposition maneuver (Aug_Man), pedicle screws augmented after the reposition maneuver (Man_Aug) showed a significant higher number of load cycles until failure. Pedicle screws augmented after the reposition maneuver (Man_Aug) had a smaller per cycle motion during loading than pedicle screws augmented prior to the reposition maneuver (Aug_Man). The predominant loosening mechanism for pedicle screws augmented after the reposition maneuver (Man_Aug) was pedicle screw toggling with the attached cement cloud within the trabecular bone. While pedicle screws augmented prior to the reposition, maneuver (Aug_Man) showed a motion of the screw within the cement cloud. However, the loosening mechanism for the Aug_Man group might also show predominantly a loosening with the cement cloud attached to the screw if the cement is fully cured during the reposition maneuver.

Some studies have compared the pull-out strength of the screws with the timing of PMMA application. Flahiff et al. compared the axial pull-out strength of pedicle screws inserted in "soft," "doughy," or "hard" PMMA cement in synthetic bone [21]. Screw placement in "doughy" PMMA resulted in the highest increase in pull-out forces, while placement in the hard cement had the smallest effect compared to the non-augmented control group. Linhardt et al. showed a more pronounced increase in axial pull-out strength for screws placed immediately after cement injection in doughy cement than for placement of the screws $60 \mathrm{~min}$ after cement injection [22]. For screw placement in doughy cement, the failure occurred at the bone-cement interface, while for screw placement after 60 min of cement injection, it occurred at the screw-cement interface. This might be due to better bonding of the cement with the screw threads for immediate screw placement in doughy cement. The failure mode of screw placement in doughy cement at the bone-cement interface is comparable to the Man_Aug group of the present study. The screw was also surrounded by doughy cement, fully bonded to the cement, and predominantly showed a failure at the cement-bone interface with the screw bonded to the cement toggling within trabecular bone. In contrast to this in the Aug_Man group, the cement-screw bonding was likely disrupted by the reposition maneuver and caused a loosening at the cement-screw interface with motion of the screw within the cement cloud still rigidly fixed to the trabecular bone.

However, these studies used a pull-out test instead of cyclic cranio-caudal loading and did not investigate the effect of cement application timing in combination with a reposition maneuver a reposition maneuver on screw anchorage.

In order not to further compromise pedicle screw anchorage in patients with reduced bone quality it is recommended to avoid correction maneuvers based on the instrumentation in patients with reduced bone quality [23]. During a reposition maneuver in combination with screw augmentation the screw anchorage can be compromised and cause clinical failure of the instrumentation by disengagement of the screw from the PMMA cement with the cement still attached to the bone [24]. To avoid compromising the screw anchorage, screws can be augmented after the reposition maneuver or in cases with augmentation prior the reposition maneuver sufficient time should be taken to allow the cement to fully cure.

The carried out reposition maneuver was standardized and intended to simulate a fracture reposition with a pivot point close to the pedicle. Due to varying lever arm ratios in reposition tools and techniques, forces measured during the simulated reposition maneuver cannot be directly transferred to clinical practice. It might be estimated that to obtain comparable reposition forces on the pedicle screw with various reposition instruments used in clinical practice only $20-50 \%$ of the forces reported with the present setup have to be applied by the surgeon to the reposition instrument.

A limitation of the study is that time points for the reposition maneuver after the augmentation were not 
varied and therefore an adequate waiting time for the reposition maneuver after augmentation can not be stated. The timespan was chosen similar to the clinical setting to achieve a good transferability into clinical practice. However, the required waiting time will likely vary depending on the brand and manufacturer of the PMMA cement.

Another limitation in this study is that it was conducted on cadaver specimens, with their inherent limitations. Cadaver specimens cannot replicate biological factors such as bone remodeling around PMMA cement. Osseous resistance, loading and in vivo loading of structures can only be approximated.

This study's strengths are that its findings underline for the first time, that the time of cement application has an effect on pedicle screw anchorage in the osteoporotic vertebral body under physiologic related cyclic craniocaudal loading [2, 25-29] and it applies a stepwise increasing load protocol designed to investigate implant anchorage in reduced bone quality $[30,31]$.

\section{Conclusions}

The time of cement application has an effect on pedicle screws anchorage in the osteoporotic vertebral body. Pedicle screws augmented after the reposition maneuver showed a significant higher number of load cycles and load magnitude until screw loosening. In summary, if a pedicle screw got loose during a reposition maneuver, cement augmentation can reinforce the pedicle screw purchase again and early screw loosening during cyclic loading might be prevented.

Acknowledgements The authors wish to thank individuals who donated their bodies and tissues for the advancement of education and research.

\section{Compliance with ethical standards}

Conflict of interest Laboratory costs were supported by Biomet Europe.

Open Access This article is distributed under the terms of the Creative Commons Attribution 4.0 International License (http://crea tivecommons.org/licenses/by/4.0/), which permits unrestricted use, distribution, and reproduction in any medium, provided you give appropriate credit to the original author(s) and the source, provide a link to the Creative Commons license, and indicate if changes were made.

\section{References}

1. Hernlund E, Svedbom A, Ivergard M, Compston J, Cooper C, Stenmark J, McCloskey EV, Jonsson B, Kanis JA (2013) Osteoporosis in the European Union: medical management, epidemiology and economic burden. Arch Osteoporos 8:136. doi:10.1007/s11657-013-0136-1

2. Bostelmann R, Keiler A, Steiger HJ, Scholz A, Cornelius JF, Schmoelz W (2015) Effect of augmentation techniques on the failure of pedicle screws under cranio-caudal cyclic loading. Eur Spine J. doi:10.1007/s00586-015-3904-3

3. Choma TJ, Pfeiffer FM, Swope RW, Hirner JP (2012) Pedicle screw design and cement augmentation in osteoporotic vertebrae: effects of fenestrations and cement viscosity on fixation and extraction. Spine (Phila Pa 1976) 37:E1628-E1632. doi:10.1097/ BRS.0b013e3182740e56

4. Elder BD, Lo SF, Holmes C, Goodwin CR, Kosztowski TA, Lina IA, Locke JE, Witham TF (2015) The biomechanics of pedicle screw augmentation with cement. Spine J 15:1432-1445. doi:10. 1016/j.spinee.2015.03.016

5. Renner SM, Lim TH, Kim WJ, Katolik L, An HS, Andersson GB (2004) Augmentation of pedicle screw fixation strength using an injectable calcium phosphate cement as a function of injection timing and method. Spine (Phila Pa 1976) 29:E212-E216

6. Tan JS, Bailey CS, Dvorak MF, Fisher CG, Cripton PA, Oxland TR (2007) Cement augmentation of vertebral screws enhances the interface strength between interbody device and vertebral body. Spine (Phila Pa 1976) 32:334-341. doi:10.1097/01.brs. 0000253645.24141 .21

7. Zhuang XM, Yu BS, Zheng ZM, Zhang JF, Lu WW (2010) Effect of the degree of osteoporosis on the biomechanical anchoring strength of the sacral pedicle screws: an in vitro comparison between unaugmented bicortical screws and polymethylmethacrylate augmented unicortical screws. Spine (Phila Pa 1976) 35:E925-E931. doi:10.1097/BRS.0b013e3181c5fb21

8. McHanwell S, Brenner E, Chirculescu ARM, Drukker J, van Mameren H, Mazzotti G, Pais D, Paulsen F, Plaisant O, Caillaud MM, Laforet E, Riederer BM, Sanudo JR, Bueno-Lopez JL, Donate-Oliver F, Sprumont P, Teofilovski-Parapid G, Moxham BJ (2008) The legal and ethical framework governing Body Donation in Europe-a review of current practice and recommendations for good practice. Eur J Anat 12:1-24

9. Riederer BM, Bolt S, Brenner E, Bueno-Lopez JL, Circulescu ARM, Davies DC, DeCaro R, Gerrits PO, McHanwell S, Pais D, Paulsen F, Sendemir E, Stabile I, Moxham BJ (2012) The legal and ethical framework governing Body Donation in Europe-1st update on current practice. Eur J Anat 16:1-21

10. Chin KR, Newcomb AG, Reis MT, Reyes PM, Hickam GA, Gabriel J, Pencle FJ, Sung RD, Crawford NR (2016) Biomechanics of posterior instrumentation in L1-L3 lateral interbody fusion: pedicle screw rod construct vs. transfacet pedicle screws. Clin Biomech (Bristol, Avon) 31:59-64. doi:10.1016/j.clin biomech.2015.10.001

11. Wilke HJ, Kaiser D, Volkheimer D, Hackenbroch C, Puschel K, Rauschmann M (2016) A pedicle screw system and a lamina hook system provide similar primary and long-term stability: a biomechanical in vitro study with quasi-static and dynamic loading conditions. Eur Spine J 25:2919-2928. doi:10.1007/ s00586-016-4679-x

12. Aycan MF, Tolunay T, Demir T, Yaman ME, Usta Y (2017) Pullout performance comparison of novel expandable pedicle screw with expandable poly-ether-ether-ketone shells and cement-augmented pedicle screws. Proc Inst Mech Eng $\mathrm{H}$ 231:169-175. doi:10.1177/0954411916687792

13. Wu ZX, Gong FT, Liu L, Ma ZS, Zhang Y, Zhao X, Yang M, Lei W, Sang HX (2012) A comparative study on screw loosening in osteoporotic lumbar spine fusion between expandable and conventional pedicle screws. Arch Orthop Trauma Surg 132:471-476. doi:10.1007/s00402-011-1439-6

14. Schroder C, Nguyen M, Kraxenberger M, Chevalier Y, Melcher C, Wegener B, Birkenmaier C (2016) Modification of PMMA 
vertebroplasty cement for reduced stiffness by addition of normal saline: a material properties evaluation. Eur Spine J. doi:10.1007/ s00586-016-4845-1

15. Schulte TL, Keiler A, Riechelmann F, Lange T, Schmoelz W (2013) Biomechanical comparison of vertebral augmentation with silicone and PMMA cement and two filling grades. Eur Spine J 22:2695-2701. doi:10.1007/s00586-013-2908-0

16. Hoppe S, Keel MJ (2017) Pedicle screw augmentation in osteoporotic spine: indications, limitations and technical aspects. Eur J Trauma Emerg Surg 43:3-8. doi:10.1007/s00068-016-0750-x

17. Pinera AR, Duran C, Lopez B, Saez I, Correia E, Alvarez L (2011) Instrumented lumbar arthrodesis in elderly patients: prospective study using cannulated cemented pedicle screw instrumentation. Eur Spine J 20(Suppl 3):408-414. doi:10.1007/ s00586-011-1907-2

18. Kohlhof H, Seidel U, Hoppe S, Keel MJ, Benneker LM (2013) Cement-augmented anterior screw fixation of Type II odontoid fractures in elderly patients with osteoporosis. Spine J 13:1858-1863. doi:10.1016/j.spinee.2013.06.031

19. Muller F, Fuchtmeier B (2013) Percutaneous cement-augmented screw fixation of bilateral osteoporotic sacral fracture. Unfallchirurg 116:950-954. doi:10.1007/s00113-013-2387-0

20. Pingel A, Kandziora F, Hoffmann CH (2014) Osteoporotic L1 burst fracture treated by short-segment percutaneous stabilization with cement-augmented screws and kyphoplasty (hybrid technique). Eur Spine J 23:2022-2023. doi:10.1007/s00586-0143496-3

21. Flahiff CM, Gober GA, Nicholas RW (1995) Pullout strength of fixation screws from polymethylmethacrylate bone cement. Biomaterials 16:533-536

22. Linhardt O, Luring C, Matussek J, Hamberger C, Plitz W, Grifka J (2006) Stability of pedicle screws after kyphoplasty augmentation: an experimental study to compare transpedicular screw fixation in soft and cured kyphoplasty cement. J Spinal Disord Tech 19:87-91. doi:10.1097/01.bsd.0000177212.52583.bd
23. Bradford DS, Tay BK, Hu SS (1999) Adult scoliosis: surgical indications, operative management, complications, and outcomes. Spine (Phila Pa 1976) 24:2617-2629

24. Mesfin A, Komanski CB, Khanna AJ (2013) Failure of cementaugmented pedicle screws in the osteoporotic spine: a case report. Geriatr Orthop Surg Rehabil 4:84-88. doi:10.1177/ 2151458513500787

25. Goel VK, Winterbottom JM, Weinstein JN (1994) A method for the fatigue testing of pedicle screw fixation devices. J Biomech 27:1383-1388

26. Kiner DW, Wybo CD, Sterba W, Yeni YN, Bartol SW, Vaidya R (2008) Biomechanical analysis of different techniques in revision spinal instrumentation: larger diameter screws versus cement augmentation. Spine (Phila Pa 1976) 33:2618-2622. doi:10.1097/ BRS.0b013e3181882cac

27. Tan JS, Kwon BK, Dvorak MF, Fisher CG, Oxland TR (2004) Pedicle screw motion in the osteoporotic spine after augmentation with laminar hooks, sublaminar wires, or calcium phosphate cement: a comparative analysis. Spine (Phila $\mathrm{Pa}$ 1976) 29:1723-1730

28. Rohlmann A, Bergmann G, Graichen F (1999) Loads on internal spinal fixators measured in different body positions. Eur Spine $\mathrm{J}$ 8:354-359

29. Rohlmann A, Graichen F, Bergmann G (2000) Influence of load carrying on loads in internal spinal fixators. J Biomech 33:1099-1104

30. Unger S, Erhart S, Kralinger F, Blauth M, Schmoelz W (2012) The effect of in situ augmentation on implant anchorage in proximal humeral head fractures. Injury 43:1759-1763. doi:10. 1016/j.injury.2012.07.003

31. Windolf M, Maza ER, Gueorguiev B, Braunstein V, Schwieger K (2010) Treatment of distal humeral fractures using conventional implants. Biomechanical evaluation of a new implant configuration. BMC Musculoskelet Disord 11:172. doi:10.1186/14712474-11-172 